\title{
Collaboration and Application Integration: Distributed Design with Virtual CAD
}

\author{
P. Bertok \\ Department of Computer Science, Royal Melbourne Institute of Technology, Australia \\ Em: pbertok@rmit.edu.au
}

J.P.T. Mo, S. Woodman

CSIRO Manufacturing Science and Technology, Australia

Keywords CAD, Application integration

\begin{abstract}
The pervasiveness of computer networking and rapid progress in computer performance have made global cooperation of designers a real possibility. While these technologies provide the hardware infrastructure, software development is struggling to keep pace with these developments. There are many legacy applications and competing new developments, but unfortunately most of those operate on specific platforms in isolated environments. Cooperation of designers working on those systems is very difficult; a significant problem being that different data converter facilities are needed to exchange, interpret and combine designs components produced on different systems. The system presented in this paper aims at integrating different CAD systems and achieve true interoperability, where components designed in one system can be easily viewed, modified or integrated into other designs. The approach taken was creating an integration platform, and different CAD systems are integrated into a virtual CAD system in a seamless manner. The original CAD systems store objects designed locally on that system, while remote access is provided via an integration layer.
\end{abstract}

\section{INTRODUCTION}

Traditionally design has been a cooperative activity, but early CAD systems were designed for single users and without sharing information with other systems. Later CAD systems became more open as they provided interfaces for data exchange to other programs and utilities, and multiuser operation has also become possible. While these developments already 
allowed designer cooperation, system heterogeneity still remains a problem. As there are many CAD systems in use, it is quite common in large scale global projects involving several design teams around the world that different groups use different CAD systems [1]. Solving the problem of designer cooperation is becoming more and more urgent. While global connectivity is already a reality, the infrastructure to connect any computer to any other computer in the world exists; its use in cooperative work is still in its infancy. This paper addresses this issue by proposing a common user interface that can access different CAD systems and can use their native CAD model data. In essence, the system described here was developed to facilitate unambiguous information flow in engineering projects.

\section{THE VIRTUAL CAD SYSTEM}

The primary objective of the "Virtual CAD" project is to develop a system that allows remote users around the world to access CAD models the same way as local users do, even though the design data may be stored in different CAD systems in different formats. The Virtual CAD system was to offer a unified interface to the user and add a convenient remote design facility while retaining useful CAD features.

Additional functionality was implemented in the unified interface, and retaining the original features was achieved by integrating the original CAD systems without any modifications. All components were integrated by using special interfacing, or wrapping software, and common utilities provided a uniform appearance. In the process existing software was used wherever possible, which included the integration platform as well. Several methods and systems were considered as integration platform, and the selection was based on user requirements, which included the required services and functionality, the preferred user interface and the user environment.

First a world-wide-web based solution was considered. Web-browsers are available under most platforms, provide convenient easy-to-use user interfaces and can display arbitrary data types by using plug-ins, hence enabling component based integration. A web-based model viewer could be easily implemented and could provide an easily expandable platform by adding new plug-ins for new model data types. The real shortcoming of a web-browser-based solution was manifested in handling modifications to design data. While data of significant complexity, e.g. graphical or even audio data, can be transferred from server to client, transferring data from client to server is restricted primarily to textual information e.g. via CGI scripts. The Virtual CAD system was intended to provide an active front-end 
at the user sites and allow users to modify different design data, however, changing design data via textual data input is very cumbersome. Based on this evaluation, the idea of web-based integration was abandoned at a very early stage.

Next, platforms providing integration on the procedure level were examined. Procedure-level integration enabled a more versatile architecture that included a user interface providing design data manipulation. This meant accessing procedures in the individual CAD systems, and also adding procedures for remote data display and manipulation. An aim was to integrate existing CAD systems, so that the Virtual CAD system will include their capabilities and enhance them.

Distributed object technology (DOT) has been widely accepted as enabling technology for distributed software improvement and re-use [2]. DOT has been used to create middleware, a middle layer software between components such as clients and servers. Middleware offers an integration platform for diverse applications, including legacy systems. DOT also uses encapsulation technique to preserve the original essence of the legacy system and to provide an external interface. The encapsulation approach has become a widely applied method and is known as wrapping.

When selecting a platform, CORBA and the Distributed Computing Environment (DCE) were looked at. CORBA soon became the final choice, as CORBA offered a real object oriented framework with many services available. In addition, the implementation chosen (Orbix) was available under the operating systems in the user environment, making the implementation work clear and straightforward.

\section{CORBA}

The Common Object Request Broker Architecture (CORBA) is a one of the most commonly used middleware. It provides an object-oriented framework that provides a universal communication infrastructure supporting a variety of object mechanisms. CORBA was designed to deal with heterogeneous components and to integrate them into cohesive distributed systems. CORBA suits client-server architectures in particular, clients being the consumers of resources provided by servers. In CORBA, as in any object-oriented system, every entity in a running program is viewed as an object with a message-handling interface. The interface specifies the behaviour of the object and it is kept separate from the implementation. Objects are encapsulated and the implementation is usually hidden from the user/client, which enables modifications or replacement of the implementations behind the interfaces. 
CORBA achieves interoperability through language independence. CORBA defines a special interface definition language (IDL) that has mappings into every major programming language. Object interfaces describe operations and associated attributes in IDL. They translate functionality offered by the server into object-oriented specifications required by the object management system. An interface described in IDL constitutes a contract between client and server, both client and server use the same interface definition to construct the executable code.

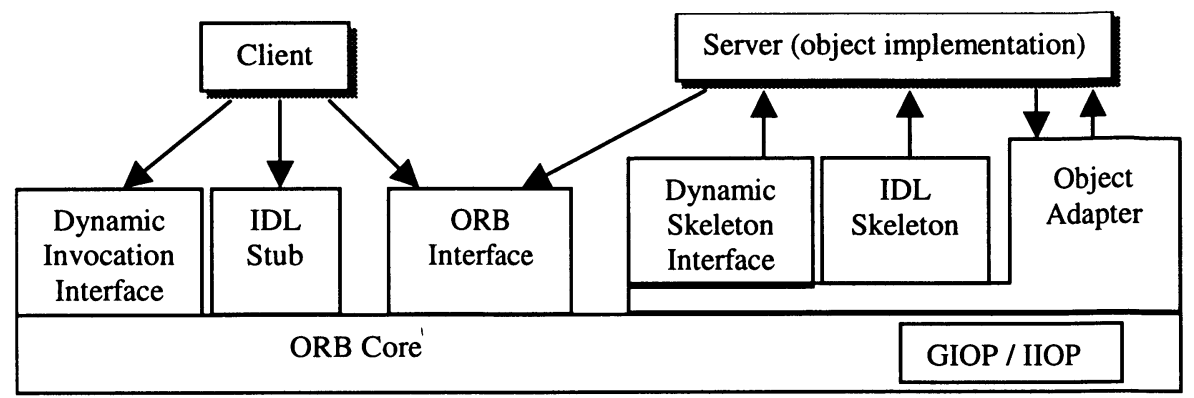

Figure 1 Common Object Request Broker Architecture

The most essential components of a CORBA system are the Object Request Brokers (ORB), which provide a communication bus connecting the different components, as shown in Figure 1. ORBs provide a mechanism to invoke a method on a remote object: an ORB locates the object, activates it if necessary and communicates the client's request to the object. ORBs support static and dynamic invocation interfaces. With static invocation the client knows the server's interface beforehand, while with dynamic invocation the client learns the server's interface through inquiries during run-time.

Interoperability of ORBs is supported by inter-ORB protocols. The General Inter-ORB Protocol (GIOP) has been developed to allow communication between independently developed software modules, without explicitly identifying the underlying network. GIOP works over any transport protocol that satisfies a minimum set of requirements. The Internet Inter-ORB protocol (IIOP) uses GIOP with TCP/IP protocol stack for transport, which is more restrictive but has beccme very popular due to the pervasiveness of the Internet.

To support different object domains CORBA defines the interoperable object reference (IOR). IORs are used when crossing object reference domain (ORB) boundaries, as there are no general rules for object implementation, every ORB can implement an object reference in a way that is the most convenient locally. 
CORBA was designed with interoperability in mind, and provides an excellent platform for integration. Independently developed components can be easily connected into one system, and it is especially suited to the integration of legacy systems into a collaborative system.

\section{DESCRIPTION OF THE VIRTUAL CAD SYSTEM}

\subsection{System Architecture}

The task described in this paper consisted of integrating a commonly used CAD system, AutoCAD, into a larger, CORBA based system, as illustrated in Figure 2. Other CAD systems, such as Pro-Engineer, SolidWorks, IDEAS, are also being considered for future integration. The clients need not be co-located, they can be distributed around the world, and each of them is running the same code, albeit with possibly slightly different local configuration data.

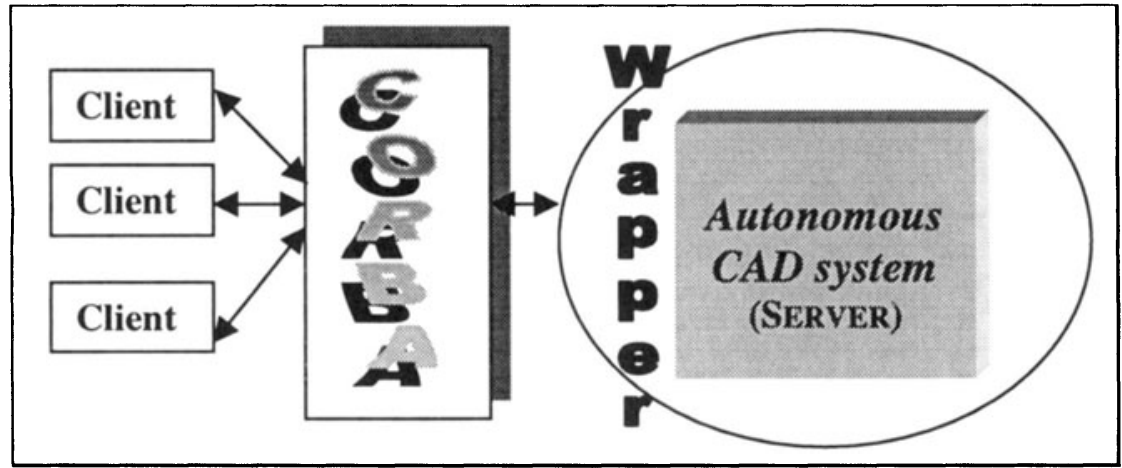

Figure 2 Wrapper Facade Pattern Applied to Virtual CAD

As different clients need access to different CAD functions and data, the so-called Component-First based wrapping approach was used [3]. This approach employs the legacy system by using its modules and application logic in the new environment. System architecture, functionality and interfaces, however, are following the new system design, so the existing logic and code are seamlessly integrated into the new environment.

The work involved a feasibility study that also examined the appropriateness of messages and data transfer. The aim was to make best use of the legacy CAD system's capabilities and of the available bandwidth in the communication network (Internet or Intranet). There was no clear oneto-one correspondence between the services/data provided by the legacy system and those required via the new client interface. However, since 
developing the client was also part of the project, it was possible to find a quasi-optimal mapping of objects between the old and new systems, which did not require too complex conversions of functions and data; in mathematical terms a many-to-many mapping system was established.

The extensibility of the wrapper was also considered, as modifications, such as including additional functions in the new interface and including other CAD systems, may need to be incorporated at a later stage. Different wrapping patterns were examined, and a Wrapper Façade [4] was eventually implemented, as it provided a simple, extendable structure that implemented many-to-many mapping.

\subsection{Implementation}

The implementation was divided into two parts, the first one laying down the class foundations and the second one addressing error handling and exceptions.

\subsubsection{Clustering functions and data structures into classes}

Within the wrapper class several classes were defined to reflect the new user interface and functionality. In many cases, the client was specified to ease the burden of interface conversion, and the local server functions are replicated at the client side. In these cases simple message passing between the two sides was sufficient. Great help was the Java 3D based client interface that could emulate many of the CAD server's 3D-manipulation capabilities [5]. Classes representing these functions simply forward their method invocations to the underlying low-level server functions.

An important feature of the Virtual CAD system is its ability to manipulate CAD primitives and modify CAD models. This is illustrated in Figure 3: the class BasicPrimitive defines common interfaces required by a CAD primitive. It associates with the TransformGroup and BranchGroup classes defined in the Java 3D library. It defines basic interfaces to transform the geometry of a primitive. In addition, this class defines two methods (post-order and pre-order) to traverse this structure. The class LeafPrimitive can define a primitive such as standard shapes and CAD models represented in neutral formats. The class CompositePrimitive can define a primitive that contains other primitives (defined as children) such as CompositePrimitive or LeafPrimitive objects. The class CompositePrimitive provides interfaces to add and delete children.

These basic primitives support the fundamental user operations in the Virtual CAD environment. For more complex operations, such as 
assembling a few components in the CAD server, additional levels of indirection are required. A possible way to implement additional indirection is dynamical dispatching of wrapper facade method implementations, which can also increase extensibility. Aggregated functions need to be developed and wrapped in the server by using languages associated with the CAD system.

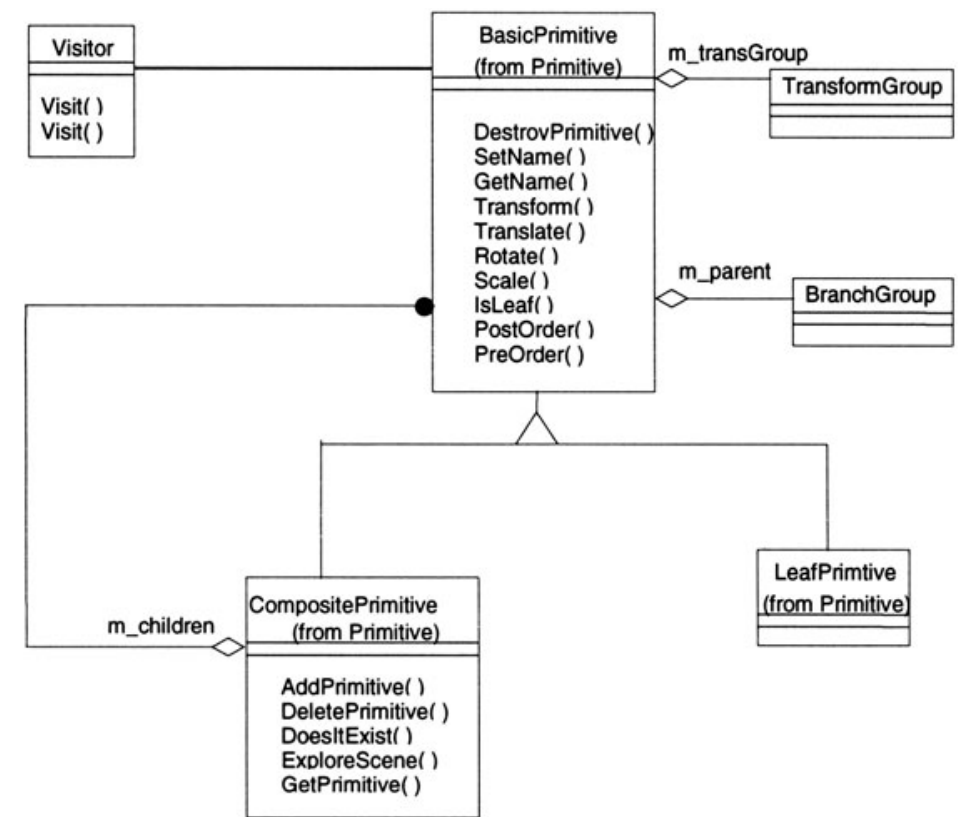

Figure 3 System Structure with CAD Object Manipulating Primitives

\subsubsection{Establishing error reporting mechanisms}

Handling errors is essential in any application. It can be kept simple by reporting back to the caller only, or can be quite complex when corrective actions may need to be initiated, and the pattern used may need to accommodate that.

In this project it was kept fairly simple. The options were the following. Errors could be communicated back to caller via standard error codes, as done on many conventional platforms. Using exception-handling mechanisms provided by CORBA was another option, but it was more complex and needed to be justified. Currently the exception handling facilities in Java on the client side are used, which provide a comprehensive set of functions for contingencies. In future the error may be communicated back to server. The error handling capabilities of the server will also have to be considered, as the server may be coded in a language different to the client. 


\section{CONCLUSION}

This paper presented a case of a virtual CAD system. The most important features of the system were the following:

- It can integrate different, common-off-the-shelf (COTS) CAD systems into one large virtual CAD system, and provides a seamless data flow from one CAD subsystem into another.

- It provides a common interface to all different CAD systems integrated into virtual-CAD.

- It can operate in a globally distributed environment.

The system was implemented using distributed object technology on a CORBA platform, with the following characteristics.

- Procedures in the individual CAD systems were accessible from the virtual CAD system.

- Implementing the Wrapper Façade software pattern allowed a simple, extendable structure that suited the application environment well.

- The program classes reflected interface and functionality.

- Message passing between client and server was the main form of communication, albeit direct remote method invocations were also necessary in a few cases.

- Error reporting was kept simple, and was implemented in form of messages sent back to the client.

\section{REFERENCES}

[1] Fowler S. and Karinthi R. (1996). Remote access to CAD databases using an information sharing system, Computers in Industry, Vol.29, 1996, pp.117-122.

[2] Mowbray T.J. and Zahavi R. (1995). The Essential CORBA-Systems Integration Using Distributed Objects, John Wiley \& Sons Inc. 1995

[3] Morin T. (1999). Migrating Legacy Systems to CORBA, http://developer.netscape.com:80/viewsource/morin_corba/morin_corba.html, 1999

[4] Schmidt D. (1999). Wrapper Facade, C++ Report, February 1999

[5] Chan P. and Lee R. (1998). The Java Class Libraries. Second Edition, Volume 2, Addison-Wesley, 1998 